\section{A eficácia da adaptação de prótese auditiva na redução ou eliminação do zumbido}

\section{The efficacy of hearing aids on diminishing or eliminating tinnitus}

\author{
Lívia Oliveira Salgueiro de Moura', Maria Cecília \\ Martinelli lório², Marisa Frasson de Azevedo ${ }^{3}$
}

Resumo / Summary

zumbido é uma desordem extremamente freqüente em pacientes com perda auditiva, atingindo cerca de 40 milhões de pessoas nos EUA, afetando aproximadamente $1 / 3$ da população acima dos 65 anos de idade. A prática clínica tem demonstrado que pacientes portadores de perda auditiva associada a zumbido beneficiam-se com o uso de próteses auditivas, pois estas, além de melhorarem a compreensão da conversação, aliviam o zumbido. Objetivo: No presente trabalho, procurou-se verificar a eficiência da adaptação de próteses auditivas na redução ou eliminação do zumbido em pacientes com perda auditiva. Forma de estudo: Estudo de série. Material e Método: Foram avaliados 47 indivíduos adultos, sendo 32 do sexo feminino e 15 do sexo masculino, com idades entre 32 e 88 anos, com indicação médica para adaptação de prótese auditiva. Realizou-se análise e avaliação subjetiva da sensação de zumbido por meio da técnica da acufenometria, caracterizando-o quanto ao pitch e loudness, e acompanhamento dos pacientes durante um ano afim de verificar se houve melhora do zumbido após a adaptação da prótese auditiva. Resultados: A maioria dos pacientes $(87,2 \%)$ referiu melhora do zumbido com o uso da prótese auditiva, sendo que em $51 \%$ destes o zumbido desapareceu completamente. 0 tempo de uso da prótese necessário para a melhora do zumbido foi de 3 a 8 meses para a maioria dos indivíduos. As características da prótese auditiva, como modelo, tecnologia e ventilação, não influenciaram a melhora do zumbido. Conclusão: A adaptação de próteses auditivas mostrou-se eficaz na redução ou eliminação do zumbido em pacientes portadores de perda auditiva associada a zumbido.
Palavras-chave: zumbido, perda auditiva, prótese auditiva. Key words: tinnitus, hearing loss, hearing aids.

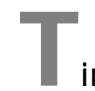

innitus is a disorder extremely frequent in patients with hearing loss. It affects more than 40 milion people in the USA, affecting about $1 / 3$ of the population above 65 years old. Clinical experience has been demonstrating that patients with hearing loss and tinnitus have great benefits with the hearing aid because it helps out the comprehension of conversation while relieves tinnitus. Aim: In this work, we have studied the efficacy of hearing aids on diminishing or eliminating tinnitus in patients with hearing loss. Study design: Series study. Material and Method: We evaluated 47 patients (32 women and 15 men) with ages ranging from 32 to 88 years old and medical indication for fitting a hearing aid. We subjectively investigated the sensation of tinnitus matching frequency $(\mathrm{Hz})$ and loudness $(\mathrm{dB})$ through the technic of acuphenometry and we have attended the patients for one year aiming to verify if there was a relief of tinnitus after the hearing aid fitting. Results: The majority of patients $(87,2 \%)$ refered relief of tinnitus with the use of the hearing aid. In $51 \%$ of these patients tinnitus has completely desapeared. It was necessary to use the hearing aid for some months (3-8) to obtain the relief of tinnitus in the majority of patients. The characteristics of the hearing aids, as model, tecnology and vent, had no influence on the relief of tinnitus. Conclusions: The use of the hearing aid was effective in reducing or eliminating tinnitus in patients with hearing loss and tinnitus.

${ }^{1}$ Fonoaudióloga. Mestre em Distúrbios da Comunicação Humana pela Universidade Federal de São Paulo- Escola Paulista de Medicina. 2 Fonoaudióloga. Professora em Distúrbios da Audição da Universidade Federal de São Paulo- Escola Paulista de Medicina. Doutora em Distúrbios da Comunicação Humana pela Universidade Federal de São Paulo- Escola Paulista de Medicina.

${ }^{3}$ Fonoaudióloga. Professora Adjunto IV do Curso de Fonoaudiologia da Universidade Federal de São Paulo- Escola Paulista de Medicina. Doutora em Distúrbios da Comunicação Humana pela Universidade Federal de São Paulo- Escola Paulista de Medicina.

Trabalho realizado no Departamento de Fonoaudiologia da Universidade Federal de São Paulo- Escola Paulista de Medicina.

Endereço para correspondência: Lívia Oliveira Salgueiro de Moura - Rua Botucatu, 221, apto 82 Vila Clementino São Paulo SP $04023-060$. Tel (0xx11) 5571-6450/ 8288-6467 - E-mail: livia_moura28@hotmail.com

Tese de Mestrado apresentada em 2004 pela Universidade Federal de São Paulo- Escola Paulista de Medicina. Artigo recebido em 29 de janeiro de 2004. Artigo aceito em 19 de julho de 2004. 


\section{INTRODUÇÃO}

O zumbido representa um enorme desafio para clínicos e pesquisadores. É uma desordem extremamente freqüente, atingindo cerca de 40 milhões de pessoas nos EUA, das quais 10 milhões são seriamente afetadas por ele. 0 zumbido afeta aproximadamente $1 / 3$ da população acima dos 65 anos de idade. $A$ atitude comum de vários profissionais é mandar 0 paciente para casa com o conselho de que "nada pode ser feito e você terá que aprender a conviver com seu zumbido"1.

Diversos autores concordam com o fato de que 0 zumbido envolve anormalidades tanto da função coclear quanto do processamento dos sinais relacionados a ele no sistema nervoso central. Além disso, o zumbido não deve ser visto somente como uma percepção de sinais originados de um gerador isolado, localizado ${ }^{2-4}$.

Segundo Salvi et al. ${ }^{3}$, um decréscimo de estímulo auditivo, mesmo sendo temporário, resulta num crescimento de sensibilidade dos neurônios dos centros subcorticais. Em certos casos, o zumbido pode ser devido a um crescimento de sensibilidade nos centros subcorticais do sistema auditivo. Assim, este pode ser tratado por um método baseado no decréscimo dessa sensibilidade.

Próteses auditivas e geradores de som (ruído branco) foram incorporados a um protocolo que emprega um aumento gradativo de estímulos auditivos por períodos prolongados de tempo. Dados no campo da Neurociência reforçam que essas mudanças plásticas requerem períodos de tempo prolongados, medidos em semanas ou meses ${ }^{4}$.

Alguns autores propõem como objetivo clínico para tratar o zumbido remover a percepção deste da consciência do paciente e iniciar e facilitar o processo de habituação ao zumbido. Esse processo envolve mudanças na força das conexões funcionais entre os neurônios envolvidos no processamento dos sinais do zumbido, com o objetivo de diminuir a detecção e a transmissão de seus sinais para os centros emocionais do cérebro ${ }^{2,4}$.

A prática clínica tem demonstrado que pacientes portadores de perda auditiva associada a zumbido beneficiam-se com o uso de próteses auditivas, pois estas, além de melhorarem a compreensão da conversação, aliviam o zumbido.

Diversos autores recomendam a adaptação de prótese auditiva em pacientes com perda auditiva acompanhada por zumbido como uma alternativa eficaz na sua melhora ${ }^{4-6}$.

Um estudo realizado por Surr et al. ${ }^{7}$ demonstrou que aproximadamente $50 \%$ dos pacientes avaliados acharam a prótese auditiva efetiva na redução ou eliminação do zumbido.

Considerando-se esses dados, o presente estudo tem por objetivo:

- verificar a eficiência da adaptação de próteses auditivas na redução ou eliminação do zumbido em pacientes portadores de perda auditiva;
- caracterizar o zumbido;

- verificar outros fatores que possam interferir na melhora do zumbido, como tempo de uso e características da prótese auditiva.

\section{MATERIAL E MÉTODO}

Foram avaliados 47 indivíduos adultos, sendo 32 do sexo feminino e 15 do sexo masculino, com idades entre 32 e 88 anos, todos com queixa de zumbido e perda auditiva, variando de grau leve a profundo, uni ou bilateral, neurossensorial, com indicação médica para adaptação de prótese auditiva.

Esses indivíduos compareceram a uma empresa de aparelhos auditivos de São José dos Campos, onde foram submetidos à anamnese detalhada, audiometria tonal liminar, logoaudiometria e seleção de prótese auditiva. Os indivíduos classificaram seu zumbido quanto ao grau de incômodo, em uma escala de 0 a 10, onde o zumbido pôde ser classificado como leve (de 0 a 4), moderado (de 5 a 7) ou severo (de 8 a 10), conforme a escala utilizada no ambulatório de zumbido do Hospital das Clínicas de São Paulo. Descrição dos procedimentos utilizados:

1. Audiometria tonal liminar: exame em cabina acústica para determinar a menor intensidade na qual o indivíduo pode detectar os tons puros. Todos os exames foram realizados no mesmo audiômetro, VSC2090-AUDITEC. O limiar de audibilidade foi tido como normal quando se encontrava até $25 \mathrm{dBNA}$; de 26 a $40 \mathrm{dBNA}$, tinha-se uma perda leve; de 41 a 70, perda moderada e; de 71 a 90 perda profunda.

2. SRT (limiar de recepção de fala): exame em cabina acústica para determinar a menor intensidade na qual o indivíduo é capaz de reconhecer $50 \%$ dos estímulos (palavras) apresentados a cada orelha.

3. Índice percentual de reconhecimento de fala (IPRF): exame em cabina acústica para verificar o reconhecimento de palavras em porcentagem de acertos, em condições ideais de ambiente acústico e intensidade do som.

4. Seleção de Prótese Auditiva (AASI): inicialmente, foi realizada a pesquisa do nível de desconforto, que consiste em exame em cabina acústica para determinar a maior intensidade sonora que o indivíduo suporta em cada orelha, com apresentação de tons puros. 0 indivíduo, então, experimentou diversos modelos de próteses auditivas, todos compatíveis com sua perda auditiva, e foi selecionado o modelo que proporcionou maior ganho e conforto ao paciente.

Os indivíduos foram submetidos à acufenometria, ou seja, tiveram pesquisados o pitch (sensação de freqüência sonora) e loudness (sensação de intensidade) do zumbido da seguinte forma: um tom puro ou um ruído de banda estreita (dependendo do tipo de zumbido relatado pelo paciente) era apresentado à orelha contralateral ao zumbi- 
do, a 15dB nível de sensação ( $d B N S$ ) em diversas freqüências, até que 0 indivíduo identificasse a freqüência mais próxima de seu zumbido, determinando assim o seu pitch (sensação de freqüência sonora). Em seguida, na freqüência estabelecida, diminuía-se a intensidade do estímulo de 5 em $5 \mathrm{~dB}$, até que 0 indivíduo identificasse a intensidade mais próxima de seu zumbido, determinando assim o seu loudness (sensação de intensidade) A partir do momento da adaptação da prótese auditiva, esses pacientes foram acompanhados (com retornos a cada 6 meses) com 0 objetivo de investigarmos, através de nova anamnese, se houve redução ou eliminação do zumbido com o uso da prótese.

Além disso, foram analisados os protocolos antigos dos pacientes que apresentaram perda auditiva associada a zumbido na ocasião da anamnese e que adquiriram prótese auditiva na época. Tais pacientes foram convidados a participar da pesquisa, sendo incluídos na amostra.

0 presente estudo foi analisado e aprovado pelo Comitê de Ética em Pesquisa da Universidade Federal de São Paulo/Hospital São Paulo sob o protocolo no 0831/02. Após esclarecimento e o consentimento dos pacientes em participar do estudo, os dados obtidos foram colocados em um protocolo de avaliação e posteriormente analisados estatisticamente, afim de investigar se 0 uso da prótese auditiva foi eficaz na redução ou eliminação do zumbido.

\section{Metodologia Estatística}

Utilizou-se o Teste de I gualdade de Duas Proporções, uma técnica paramétrica que compara se a proporção de respostas de duas determinadas variáveis e/ ou seus níveis é estatisticamente significante. Este teste foi utilizado na Caracterização da Amostra e na Caracterização do Zumbido.

O outro teste utilizado foi o TesteQui-Quadrado para Independência que é um teste não paramétrico utilizado para se verificar se duas variáveis e seus níveis possuem ou não uma dependência (associação) estatística. Este teste foi utilizado para verificar os Fatores que Interferem na Melhora do Zumbido.

Utilizou-se um nível de significância de 0,05 (5\%).

0 intervalo de confiança foi construído com $95 \%$ de confiança.

\section{RESULTADOS}

A amostra foi caracterizada quanto a: idade, grau de perda auditiva e tipo de adaptação de prótese auditiva.

A idade dos indivíduos estudados variou de 32 a 88 anos, com idade média de 65,5 (desvio padrão de 13,8 ) e mediana de 66 anos. 0 intervalo de confiança estabelecido variou de 61,6 a 69,5.

Nos resultados das audiomertrias, observou-se um predomínio da perda auditiva de grau moderado (44,6\%), não diferindo estatisticamente das perdas auditivas de grau moderadamente severo $(29,7 \%)$. As perdas auditivas de grau severo $(6,4 \%)$ e profundo $(4,3 \%)$ foram as de menor ocorrência de forma estatisticamente significante.

Quanto ao tipo de adaptação da prótese auditiva, observou-se que 40 indivíduos $(85,1 \%)$ optaram por adaptação monoaural, sendo $25(62,5 \%)$ à orelha direita e 15 $(37,5 \%)$ à esquerda. Sete indivíduos (14,9\%) optaram por adaptação binaural.

A sensação de intensidade (loudness) do zumbido da população estudada é ilustrada no Gráfico 1.

Quanto à sensação de freqüência (pitch) do zumbido, 53,2\% dos indivíduos apresentaram zumbido de pitch grave e $46,8 \%$ agudo.

A localização do zumbido da população estudada é ilustrada no Gráfico 2.

Considerando somente a localização unilateral, não houve diferença proporcional da ocorrência do zumbido em relação ao lado da orelha, sendo 7 indivíduos (63,3\%) com zumbido à orelha direita e 4 indivíduos (36,4\%) à esquerda.

Trinta e nove indivíduos (83\%) apresentaram zumbido constante, estatisticamente diferente do zumbido intermitente (17\%).

0 grau de incômodo do zumbido na população estudada é ilustrado na Tabela 1.

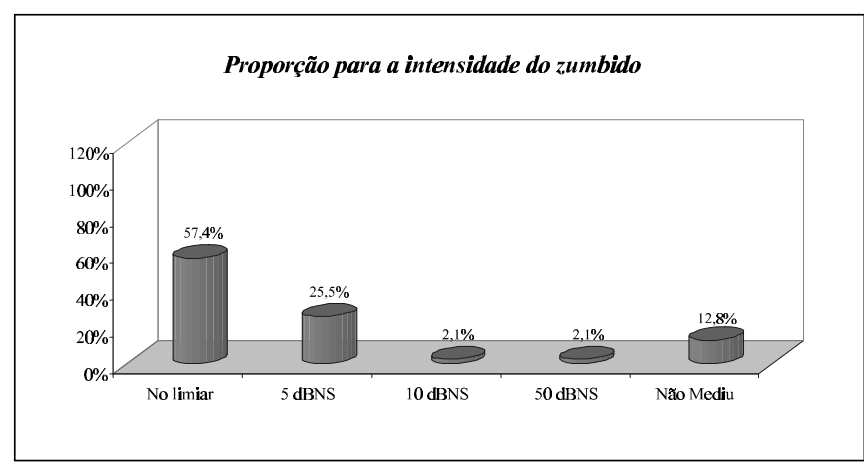

Gráfico 1. Distribuição da população em relação à sensação de intensidade (loudness) do zumbido.

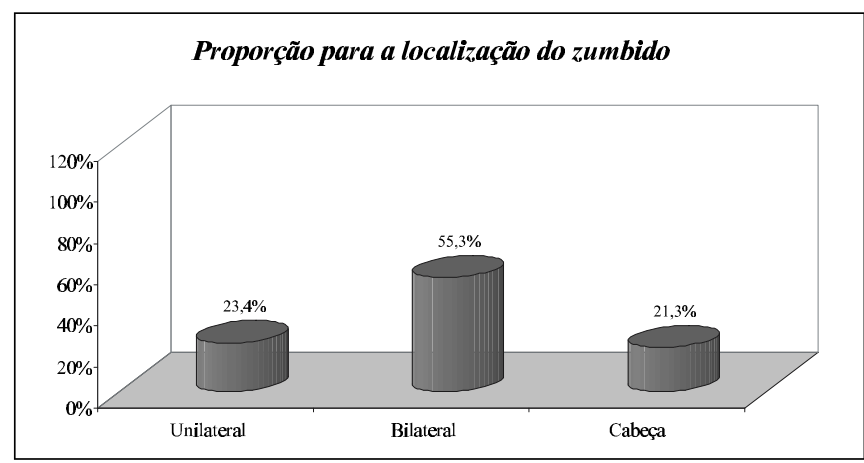

Gráfico 2. Distribuição da população em relação à localização do zumbido 
Tabela 1. Distribuição da população estudada em relação ao grau de incômodo do zumbido.

\begin{tabular}{ccc}
\hline Incômodo & $\mathrm{N}$ & $\%$ \\
\hline Leve (0-4) & 12 & $26 \%$ \\
Moderado(5-7) & 23 & $49 \%$ \\
Severo (8-10) & 12 & $26 \%$ \\
\hline Total & 47 & $100 \%$ \\
\hline Incômodo & Leve (0-4) & Moderado (5-7) \\
Moderado (5-7) & $0,019 *$ & \\
Severo (8-10) & 1,000 & $0,019 *$ \\
\hline p-valores & &
\end{tabular}

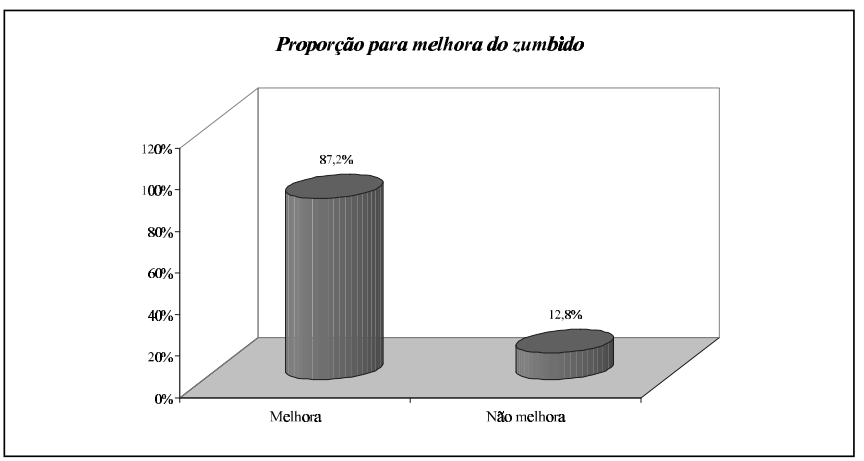

Gráfico 3. Ocorrência da melhora do zumbido com o uso da prótese auditiva

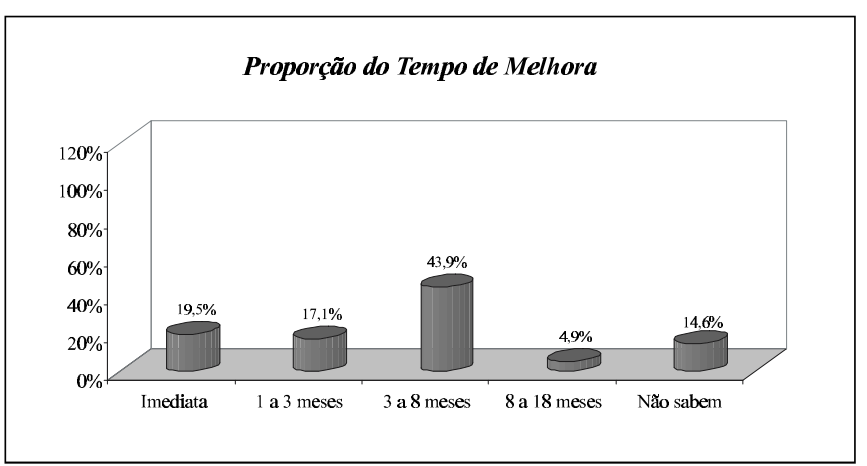

Gráfico 4. Tempo de uso da prótese necessário para a melhora do zumbido

Procurou-se verificar se haveria dependência entre 0 loudness do zumbido e o grau de incômodo referido pelo paciente. Não houve dependência estatística, no entanto 0 p-valor está muito próximo do limite aceitável, assim podemos dizer que existe uma tendência a ocorrer dependência entre a intensidade do zumbido e o grau de incômodo.

Houve melhora estatisticamente significante do zumbido com o uso da prótese auditiva, conforme ilustra o Gráfico 3.
Analisaram-se os fatores que poderiam interferir na melhora do zumbido:

- Idade do Indivíduo

- Tempo de uso da prótese auditiva

- Características da prótese auditiva (ventilação, modelo de prótese e tecnologia)

- Caracterização do zumbido (Localização, Constância, Intensidade e Freqüência)

Não houve nenhuma dependência estatisticamente significativa entre a idade dos indivíduos e a melhora do zumbido com a prótese auditiva.

Nos indivíduos que melhoraram, o tempo de uso da prótese auditiva necessário para esta melhora foi de três a oito meses para a maioria dos indivíduos $(43,9 \%)$, estatisticamente diferente dos outros perío dos de tempo. 0 Gráfico 4 ilustra o tempo de uso necessário para a melhora do zumbido nos indivíduos estudados.

Estudou-se os tipos de prótese auditiva em relação ao modelo (características estéticas). Primeiramente, verificou-se a proporção de indivíduos para cada modelo de prótese auditiva. Os modelos de próteses auditivas utilizados pelos pacientes da amostra foram: retroauricular, intraauricular (concha), intracanal e microcanal, descritos por Almeida e lório (1996). A maior parte dos indivíduos (38,3\%) utilizou o modelo intracanal. Quinze indivíduos $(31,9 \%)$ utilizaram o modelo retroauricular, 4 indivíduos (8,5\%) o modelo concha e 10 indivíduos $(21,27 \%)$ o microcanal.

Não houve associação estatisticamente significante entre a melhora do zumbido e o modelo da prótese auditiva.

Quanto à tecnologia da prótese auditiva, a analógica foi a mais empregada e esta foi estatisticamente diferente das demais tecnologias, que por sua vez foram proporcionalmente empregadas da mesma maneira. Trinta e um indivíduos $(65,9 \%)$ utilizaram prótese analógica, 10 indivíduos $(21,3 \%)$ prótese programável e 6 indivíduos $(12,8 \%)$ prótese digital.

A tecnologia da prótese auditiva não influenciou a melhora do zumbido.

A ventilação do molde esteve presente em 40 indivíduos (85,1\%). Destes, 35 indivíduos $(87,5 \%)$ obtiveram melhora do zumbido com o uso da prótese. Sete indivíduos $(14,9 \%)$ não possuíam ventilação no molde. Destes, seis $(85,7 \%)$ obtiveram melhora do zumbido com o uso da prótese. Então, a presença da ventilação não influenciou a melhora do zumbido com o uso da prótese auditiva.

Para as próteses ventiladas, o tamanho da ventilação também não influenciou a melhora do zumbido.

As características como localização, constância, sensação de intensidade e sensação de freqüência do zumbido não influenciaram sua melhora com o uso da prótese auditiva, ou seja, houve melhora do zumbido em $87,2 \%$ dos indivíduos independentemente das suas características. 


\section{DISCUSSÃO}

No presente estudo, a idade média dos indivíduos estudados foi de 65 anos, variando de 32 a 88 anos de idade, sendo a maioria portadora de perda auditiva há mais de 10 anos, ou seja, a amostra foi predominantemente idosa.

Com relação ao tipo de adaptação da prótese, 85\% dos indivíduos optaram por adaptação monoaural, embora a adaptação binaural tenha sido tentada em todos os pacientes por trazer maiores benefícios ao usuário. A opção por monoaural ocorreu devido a questões financeiras e estéticas, concordando com Vaughan-Jones et al. ${ }^{8}$, que afirmaram que o número de adaptações monoaurais costuma ser maior principalmente por questões estéticas e financeiras.

Dos 72 indivíduos que compareceram para protetização, 47 (65\%) apresentaram zumbido. Sataloff et al. ${ }^{1}$ relataram que aproximadamente $85 \%$ dos pacientes que procuram um otologista têm zumbido. Stouffer e Tyler', através da distribuição de um questionário a 528 pacientes com zumbido, concluíram que para $48 \%$ da amostra a queixa primária era zumbido e para $38 \%$ era auditiva. Benevides ${ }^{10}$ relatou que o zumbido é um sintoma que freqüentemente acompanha a presbiacusia e costuma ser mais perturbador do que a surdez. Segundo Jurca et al. ${ }^{11}$, na rotina clínica do setor de audiologia, freqüentemente aparecem indivíduos com idade acima de 60 anos, cuja queixa principal é a diminuição da audição, zumbido e prejuízo na inteligibilidade da fala.

O fato de um grande número de indivíduos com perda auditiva apresentarem zumbido pode ser explicado baseando-se nas pesquisas dos diversos auto res que estudaram a fisiologia do zumbido. Alguns hipotetizaram que 0 zumbido fosse gerado na porção da membrana basilar onde há preservação de células ciliadas internas mas danos ou disfunções temporárias de células ciliadas externas ${ }^{12,13}$. Jastreboff ${ }^{2}$ afirmou que um decréscimo de in put das células ciliadas externas pertencentes à porção da membrana basilar pode resultar num decréscimo de atividade das fibras eferentes e decréscimo da inibição das vias aferentes vindas das células ciliadas internas. Isso vai acentuar a ativação das células ciliadas internas normais, resultando numa atividade anormal percebida como zumbido. 0 autor acrescentou que praticamente qualquer modificação local das propriedades da transdução coclear pode gerar zumbido devido a um contraste acentuado entre as áreas normais e alteradas, 0 que pode ser amplificado pelo processamento central no sistema nervoso. Salvi et al. ${ }^{3}$ referiram que um decréscimo de estímulos auditivos, mesmo sendo temporário, resulta num aumento de sensibilidade dos neurônios dos centros subcorticais e Hazell e Sheldrake ${ }^{4}$ postularam que em certos casos o zumbido pode ser devido a um crescimento de sensibilidade nos centros subcorticais do sistema auditivo.

No presente estudo, realizou-se a pesquisa da sensação de freqüência (pitch) e da sensação de intensidade (loudness)do zumbido. Diversos autores enfatizaram a importância da mensuração do pitch e do loudness do zumbido para experiências clínicas de tratamentos propostos, para auxiliar 0 aconselhamento ao paciente e para diversos outros tipos de pesquisa do zumbido ${ }^{5,6,14-16 .}$. Em contrapartida, alguns autores criticam a mensuração das características do zumbido, alegando que o indivíduo avaliado pode ficar confuso e que assim os resultados não serão confiáveis ${ }^{17}$. Henry e Meikle ${ }^{16}$ citaram que na clínica, há pacientes para quem freqüências acima de $3000 \mathrm{~Hz}$ são todas similares, o que dificulta a pesquisa do pitch do zumbido. Por isso, no presente estudo, o pitch do zumbido foi classificado apenas como sendo grave ou agudo.

A sensação de intensidade (loudness) do zumbido na maioria dos indivíduos $(57,4 \%)$ coincidiu com o limiar de audibilidade, quando foi possível medi-lo pela acufenometria. A maioria dos autores da literatura pesquisada encontrou 0 loudness do zumbido apenas poucos dB acima do limiar de audibilidade $\mathrm{e}^{16,19}$. Concordando com eles, outros autores especificaram suas medidas referindo que o loudness do zumbido geralmente aparecia em níveis de apenas 5 a $10 \mathrm{~dB}$ nível de sensação (NS) ${ }^{20,21,22}$. Somente Chéry-Croze et al. ${ }^{23}$ encontraram medidas de loudn ess superiores às encontradas pelos demais autores (entre 4 e 28dBNS), em pacientes com zumbido. Mas, os mesmos autores, num estudo em 1994, evidenciaram que a sensação de intensidade mensurada pelo método ascendente de todos os indivíduos com zumbido foi encontrada entre 5 e 15dBNS.

Q uanto à sensação de freqüência (pitch) do zumbido, não houve diferença proporcionalmente significativa, sendo $53,2 \%$ grave e $46,8 \%$ agudo. Estes dados diferem da literatura pesquisada, em que a maioria dos autores referiu que geralmente o pitch do zumbido tende a ser agudo, apresentando-se acima de $3000 \mathrm{~Hz}$ na maioria dos pacientes $^{18,24}$, de $6000 \mathrm{~Hz}$ a $8000 \mathrm{~Hz}^{26}$, variando de $3100 \mathrm{~Hz}$ a $10300 \mathrm{~Hz}^{23,25}$ e de $5000 \mathrm{~Hz}$ a $10000 \mathrm{~Hz}^{17}$ para a maioria dos pacientes.

Mor $(2003)^{26}$, concordando com outros autores, encontrou o pitch do zumbido identificado como tom puro agudo em $66 \%$ dos indivíduos, principalmente nas freqüências $6000 \mathrm{~Hz}$ e $8000 \mathrm{~Hz}$, em pacientes com audição normal e queixa de zumbido.

Seis indivíduos $(12,8 \%)$ referiram que o zumbido não era semelhante a nenhum som do audiômetro, o que concorda com Olsen et al. (1996 a) ${ }^{17}$, que relataram que, para muitos pacientes, o zumbido tem um caráter tonal, para outros o som é descrito como ruído (um trem distante, cachoeira, um avião sobrevoando etc). No estudo de ChéryCroze et al.(1993) ${ }^{23}$ um indivíduo (8\%) apresentava zumbido pulsátil e não foi capaz de compará-lo a nenhuma freqüência do audiômetro. No estudo de Mor (2003) ${ }^{26}$, quatro $(13,3 \%)$ pacientes não conseguiram identificar semelhança do zumbido a nenhuma das freqüências ou intensidades apresentadas. 
A maior parte dos indivíduos estudados apresentaram zumbido de localização bilateral $(55,3 \%)$, o que concorda com Meikle e Walsh ${ }^{24}$, que referiram que, na prática, a maioria dos pacientes apresenta zumbido bilateral.

No presente estudo, em pacientes com zumbido unilateral $23,4 \%$ ), não houve diferença entre as orel has direita e esquerda, o que difere dos achados de Axelsson e Ringdah $1{ }^{27}$ e Stouffer e Tyler ${ }^{9}$ que em pesquisa com distribuição de questionários, observaram que o zumbido apareceu com maior freqüência à orelha esquerda.

Verificou-se que o zumbido constante (83\%) oi mais freqüente do que o zumbido intermitente, concordando com Stouffer e Tyler ${ }^{9}$ que obtiveram $74 \%$ dos indivíduos da amostra com zumbido freqüente ou constante, em sua pesquisa com 528 indivíduos portadores de zumbido. Já Surr et al. ${ }^{7}$ encontraram uma menor porcentagem de zumbido freqüente ou constante $(43,5 \%)$ na aplicação de um questionário a 200 novos usuários de prótese auditiva. Axelsson e Ringdah ${ }^{27}$, em pesquisa com amostra randomizada, verificaram que $14,2 \%$ dos indivíduos referiram o zumbido como freqüente ou constante. Essa menor porcentagem de zumbido freqüente ou constante poderia ser explicada pelo fato da amostra randomizada incluir pessoas com audição normal e sem queixas de zumbido.

Com relação ao grau de incômodo provocado pelo zumbido, verificou-se que o grau de incômodo moderado (de 5 a 7 na escala de severidade) foi o mais freqüente (49\%). Considerando os graus de incômodo moderado e severo, $75 \%$ dos indivíduos de amostra classificaram seu zumbido com valor acima de 5 na escala proposta. Esse dado assemelha-se aos achados de Meikle et al. ${ }^{28}$, que obtiveram informações detalhadas sobre o zumbido de 1800 pacientes de uma clínica de zumbido, observando que em mais de $90 \%$ dos casos a severidade do zumbido esteve com valor acima de 5 na escala de severidade. Concordando com eles, Sanchez e Ferrari ${ }^{29}$, que analisaram 23 pacientes com zumbido constante, encontraram $95,6 \%$ dos casos com zumbido severo ou moderado.

Entretanto, alguns autores encontraram porcentagens inferiores, como Axelsson e Ringdahl ${ }^{27}$, que referiram que $2,4 \%$ da população entrevistada apresentou zumbido severo, e Surr et al. ${ }^{7}$, que encontraram $6,5 \%$ de zumbido severo em sua amostra. Tais diferenças podem ser devido às diferentes amostras estudadas, pois, em ambos os estudos, a queixa de zumbido não foi um pré-requisito para os indivíduos participarem da amostra, diferindo assim da metodologia dos outros autores ${ }^{28,29}$ e do presente estudo, nos quais a queixa de zumbido foi pré-requisito para os indivíduos fazerem parte da amostra.

Não houve dependência estatística entre a intensidade de zumbido e o grau de incômodo que este representa para o indivíduo, no entanto o $\mathrm{p}$-valor está muito próximo do limite aceitável, assim pode-se dizer que houve uma tendência a existir dependência entre o loudness do zumbido e o grau de incômodo. Diversos estudos têm demonstrado que não tem sido possível estabelecer uma correlação consistente entre a medida do loudness e o grau de incômodo (severidade) do zumbido percebido pelo paciente ${ }^{16,17,26}$. Alguns autores encontraram correlações fracas ou moderadas entre o loudness e o grau de incômodo do zumbido ${ }^{15,30}$

Pôde-se verificar que a maioria dos pacientes apresentou zumbido bilateral constante, com loudness coincidindo com o limiar de audibilidade, semelhante aos achados da literatura pesquisada.

A maioria dos pacientes $(87,2 \%)$ referiu melhora do zumbido com o uso da prótese auditiva, sendo que em $51 \%$ destes pacientes o zumbido desapareceu completamente. 0 tempo de uso da prótese auditiva necessário para a melhora do zumbido foi de 3 a 8 meses na maioria dos indivíduos estudados. Estes achados concordam com diversos autores da literatura pesquisada. Fukuda ${ }^{6}$ referiu que a prótese auditiva está indicada nos casos em que também há hipoacusia, além do zumbido. $\mathrm{H}$ azell et al. ${ }^{5}$ afirmaram que é possível diminuir o zumbido aumentando a entrada de sons (por exemplo, utilizando aparelhos de amplificação sonora individual - prótese auditiva). Isso pode ser parcialmente explicado pelo mascaramento auditivo periférico provocado pela amplificação de sons ambientais, mas, para muitos pacientes, esse efeito não é instantâneo e pode levar dias, indicando a necessidade de considerar as mudanças plásticas do sistema nervoso central. Surr et al. ${ }^{7}$ aplicaram um questionário a 200 novos usuários de prótese auditiva, observando que aproximadamente $50 \%$ dos indivíduos com zumbido relataram que a prótese auditiva proporcionou melhora parcial ou total do zumbido, o que foi considerado um importante fator na satisfação da adaptação das próteses. Sanchez e Ferrari ${ }^{29}$ referiram que, nos casos de pacientes com zumbido severo e perda auditiva associada, as próteses auditivas podem ser uma opção para a melhora do zumbido. Em pesquisa com 23 pacientes com zumbido constante, após a adaptação das próteses auditivas as autoras obtiveram 4 casos de abolição do zumbido e 12 de melhora parcial, totalizando 16 casos de respostas favoráveis $(69,5 \%)$. Hazell e Sheldrake 4 incorporaram próteses auditivas e geradores de som (ruído branco) a um protocolo que emprega um aumento gradual de estímulos auditivos por períodos prolongados de tempo, com o objetivo de reverter o processo de reconhecimento do padrão do zumbido. Dados no campo da neurociência reforçam que essas mudanças plásticas requerem períodos de tempo prolongados, medidos em semanas ou meses, concordando com o presente estudo, em que 0 tempo de uso da prótese necessário para melhora do zumbido foi de 3 a 8 meses na maioria dos indivíduos.

Foram consideradas as preferências estéticas dos pa- 
cientes no processo de seleção da prótese auditiva. Assim, o modelo de prótese auditiva mais utilizada foi o intracanal, provavelmente por ser mais discreto do que os modelos retroauricular e concha e mais barato do que 0 modelo microcanal, concordando com Almeida e lório ${ }^{31}$ que afirmaram que as próteses intra-aurais representam $80 \%$ do total de vendas anuais nos Estados Unidos, uma vez que aliam a reabilitação auditiva a um resultado estético mais satisfatório.

Embora se saiba que a tecnologia digital proporciona melhor qualidade sonora além de maiores possibilidades de ajustes e regulagens das próteses auditivas, a tecnologia de prótese auditiva mais utilizada no presente estudo foi a analógica $(65,9 \%)$, o que pode ser explicado pelo baixo custo deste tipo de prótese auditiva. Já no estudo de Sanchez e Ferrari ${ }^{29} 0$ modelo de prótese auditiva mais utilizado foi o retroauricular, com tecnologia digital (56,5\%).

Verificou-se que as características da prótese auditiva (como modelo, tecnologia, presença da ventilação e tamanho da ventilação) não influenciaram a melhora do zumbido, discordando de Sanchez e Ferrari29, que sugerem moldes bem ventilados (ventilação maior que $2 \mathrm{~mm}$ ) para melhora do zumbido. As autoras adaptaram próteses com moldes bem ventilados em 23 pacientes com zumbido, obtendo $69,5 \%$ de melhora do zumbido. No presente estudo, $87,5 \%$ dos indivíduos com moldes ventilados apresentaram melhora do zumbido e $85,7 \%$ dos pacientes com moldes sem ventilação também apresentaram melhora, não havendo, portanto, influência da ventilação na melhora do zumbido.

Verificou-se ainda que as características do zumbido (localização, sensação de intensidade, sensação de freqüência e constância) não influenciam sua melhora com o uso da prótese auditiva.

Como principal achado do presente estudo, verificou-se que a maioria dos indivíduos $(87,2 \%)$ obteve melhora do zumbido com o uso da prótese auditiva, tendo sido necessário cerca de 3 a 8 meses de uso para tal melhora.

\section{CONCLUSÕES}

Pudemos concluir que:

1. A maioria dos indivíduos (83\%) apresentou zumbido constante, de localização bilateral $(55,3 \%)$, com sensação de intensidade (loudness) coincidindo com o limiar de audibilidade $(57,4 \%)$, sem predomínio estatisticamente significante quanto à sensação de freqüência (pitch).

2. Houve melhora estatisticamente significante do zumbido com o uso da prótese auditiva em $87,2 \%$ dos indivíduos, sendo que em $51 \%$ destes o zumbido desapareceu completamente.

3. O tempo de uso da prótese necessário para melhora do zumbido foi de três a oito meses para a maioria dos indivíduos, sendo estatisticamente significante.

4. Não houve influência das características da prótese auditiva (como modelo, tecnologia, presença de ventilação e tamanho da ventilação) na melhora do zumbido.

\section{REFERÊNCIASBIBLIOGRÁFICAS}

1. Sataloff J, Sataloff RT, Lueneburg W. Tinnitus and vertigo in healthy senior citizens without a history of noise exposure. Am J Otology 1987; 8(2): 87-9.

2. Jastreboff PJ. Phantom auditory perception (tinnitus): mechanisms of generation and perception. Neurosci Res 1990; 8:221-5.

3. Salvi RJ, Saunders SS, Gratton MA, Arehole S, Powers N. Enhanced evoked response amplitudes in the inferior colliculus of the chinchilla following acoustic trauma. Hear Res 1990; 50: 245-58.

4. Hazell JWP, Sheldrake JB. Hyperacusis and tinnitus. In: Aran JM, Dauman R, eds. Proceedings IV International Tinnitus Seminar, Bordeaux; 1991. Amsterdam: Kugler, Ghedini Publications 1992 245-8.

5. Hazell JW, Wood SM, Cooper HR, Stephens SD, Corcoran AL, Coles RR., Baskill JL, Sheldrake JB. A clinical study of tinnitus maskers. Br J Audiol 1985; 19:65-146.

6. Fukuda Y. Zumbido Neurossensorial. Rev Neurociências 2000 8(1): 6-10.

7. Surr RK, Montgomery AA, Mueller HG. Effect of amplification on tinnitus among new hearing aid users. Ear Hear 1985; 6(2):71-5.

8. Vaughan-Jones RH, Padghan ND, Christmas HE, Irwin J, Doig MA. One aid or two? More visits please! J Laryngol Otol 1993; 107(4):329-32.

9. Stouffer JL, Tyler RS. Characterization of tinnitus by tinnitus patients. Journal of Speech and Hearing Disorders 1990; 55:439-53.

10. Benevides W. Otorrinolaringologista. Rio de Janeiro: Cultura Médica; 1997.

11. Jurca APK, Pinheiro FCC, Martins KC, Herrera LF, Colleone LM, Saes SO. Estudo do perfil audiológico de pacientes com idade acima de 60 anos. Salusvita 21(1):51-8.

12. Bohne BA, Clark WW. Cochlear damage following interrupted exposure to high-frequency noise. Hear Res 1987; 29:251-64.

13. Stypulkowski PH. Physiological mechanisms of salicylate ototoxicity PhD. Thesis, University of Connecticut, Storrs, 176pp, 1989.

14. Brennan JF, Jastreboff PJ. Generalization of conditioned suppession during salicylate-induced phantom auditory perception in rats. Acta Neurobiol Exp 1991; 51:15-27.

15. Risey J, Briner W, Guth PS, Norris $\mathrm{CH}$. The superiority of the Goodwin procedure over the traditional procedure in measuring the loudness level of tinnitus. Ear Hear 1989; 10:318-22.

16. Henry JA, Meikle MB. Psychoacoustic Measures of tinnitus. J Am Acad Audiol 2000; 11:138-155.

17.OIsen SO, Nielsen LH, Osterhammel PA, Rasmussen AN. Experiências para a avaliação de zumbido usando métodos subjetivos e objetivos. Rev Brasil Atual Otorrinolaringol 1996a. 3:38-44.

18. Reed GF. An audiometric study of two hundred cases of subective tinnitus. Arch Otolaryngol Head Neck Surg 1960; 71:94-104.

19. Graham JT, Newby HA. Acoustical characteristics of tinnitus: an analysis. Arch Otolaryngol Head Neck Surg 1962; 75:82-7.

20. Fowler EP. The "illusion of loudness" of tinnitus - its etiology and treatment. Laryngoscope 1942; 52:275-85.

21. Fowler EP. Control of head noises, their illusions of loudness and of timbre. Arch otolaryngol 1943; 37:391-8.

22. Ribeiro PJ, lório MCM, Fukuda Y. Tipos de zumbido e sua influência na vida do paciente: estudo em uma população ambulatorial. Acta AWHO 2000; 19(3):125-35.

23. Chéry-Croze $S$, Collet $L$, Morgon A. Medial Olivo-cochlear system and tinnitus. Acta Otolaryngol 1993; (Stoch) 113:285-90. 
24. Meikle MB, Walsh ET. Characteristics of tinnitus and related observations in over 1800 patients. J Laryngol Otol Suppl 1984; 9:17-21.

25. Chéry-Croze S, Moulin A, Collet L, Morgon A. Is the test of medial efferent system function a relevant investigation in tinnitus? Britsh Journal of Audiology 1994a; 28:13-25.

26. Mor R. Emissões Otoacústicas e Audiometria de altas freqüências: estudo em pacientes com zumbido sem perda auditiva. [Tese de Mestrado]. São Paulo: UNIFESP 2003.

27. Axelsson A, Ringdahl A. Tinnitus- a study of its prevalence and characteristics. Br J Audiol 1989; 23(1): 53-62.
28. Meikle MB, Vernon J, Johnson RM.The perceived severity of tinnitus. Some observations concerning a large population of tinnitus clinic patients. Otolaryngol. Head Neck Surg 1984; 92: 39-44.

29. Sanchez TG, Ferrari GMS. O controle do zumbido por meio da prótese auditiva: sugestões para otimização do uso. Pró-Fono Revista de Atualização Científica 2002; 14(4):111-8.

30. Jakes SC, Hallam RC, Chambers CC, Hinchcliffe R. Matched and self-reported loudness of tinnitus: methods and sources of error. Audiology 1986; 25:92-100.

31. Almeida K, lório MCM. Próteses auditivas - fundamentos teóricos e aplicações clínicas. São Paulo: Lovise; 1996. Cap.3, p.45-74. 